\title{
Transient loading of a rapidly advancing Mode-II crack in a viscoelastic medium
}

\author{
Y.A. Antipov ${ }^{\mathrm{a}, 1}$, J.R. Willis ${ }^{\mathrm{b}, *}$ \\ a Department of Mathematical Sciences, University of Bath, Claverton Down, Bath BA2 7AY, UK \\ ${ }^{\mathrm{b}}$ Department of Applied Mathematics and Theoretical Physics, University of Cambridge, Silver Street, Cambridge CB3 $9 E W$, UK
}

Received 15 June 2001; received in revised form 13 January 2002

\begin{abstract}
The problem that is studied concerns a semi-infinite crack in an infinite viscoelastic medium, propagating at uniform speed but subject to time-dependent loading. A particular loading, consisting of a pair of concentrated shear forces which come into existence as the crack passes their location, is studied in detail. Both sub-Rayleigh and transonic speed ranges are considered. The solution displays a richer range of possible behaviour than the corresponding solution for an elastic medium, which is likely to reflect in practice on the range of propagation speeds that may be achievable.

(c) 2002 Elsevier Science Ltd. All rights reserved.
\end{abstract}

Keywords: Viscoelastic media; Dynamic fracture; Laplace transform; Riemann-Hilbert problem; Stress-intensity factor

\section{Introduction}

The background motivation for this study is provided by the experimental observations of Rosakis et al. (1999) of cracks, loaded under shear, that propagate faster than the speed of shear waves. The media are usually polymers, and implications of the phenomenon are certainly of interest for composite materials which may have weak interfaces. Therefore, some degree of viscoelastic response is likely to be observed, and it

\footnotetext{
${ }^{*}$ Corresponding author. Tel.: +44-1223-339251; fax: +441223-337918.

E-mail address: j.r.willis@damtp.cam.ac.uk (J.R. Willis).

${ }^{1}$ Present address: Department of Mathematics, Louisiana State University, Baton Rouge, LA 70803, USA.
}

becomes desirable to make a study of rapidly propagating cracks, loaded dynamically, in viscoelastic media. The present work constitutes a first step in this direction. It identifies features associated with viscoelastic response in an illustrative way through consideration of a standard linear solid with constant Poisson's ratio; detailed predictions suitable for comparison with experimental observations will be addressed in later work. A very recent contribution to the theory for an elastic medium is that of Huang and Gao (2001), who considered the transonic propagation of a crack whose faces were subjected to concentrated shear forces. The resulting solution is one from which solutions for other static loadings can be constructed, by superposition. Here, the corresponding solution is found, when the medium is a standard linear solid. Sub-Rayleigh as well as 
transonic crack speeds are considered. SuperRayleigh but sub-shear wave speed propagation is omitted, because it is known not to be possible in an elastic medium displaying cohesion (in contrast to propagation of a displacement jump along a frictional interface; see, for instance, Broberg, 1989). We demonstrate a loss of uniqueness of solution for a viscoelastic medium, in that speed range.

The general qualitative observation is, not surprisingly, that the stress ahead of the crack (which, in practice, must suffice to ensure its continued extension) is reduced, relative to its "elastic" value, by the viscoelasticity. The singular behaviour at the crack tip has the same dependence on distance from the crack tip as in the elastic case, but the coefficient of the singular term is reduced. Contrary to our prior expectations, the decay with time of the coefficient is always algebraic, never exponential. However, a long-time asymptotic analysis of the stress at arbitrary distance $\xi$ ahead of the crack shows exponential decay with $\xi$, for some but not all crack speeds. Changes in behaviour are observed as the crack speed $v$ increases through the various wave speeds. As first observed by Willis (1967) in the context of subsonic propagation under Mode III loading, these are not only the highfrequency "elastic" wave speeds, but also the speeds with which plane waves of low frequency, associated with the fully relaxed elastic moduli, may propagate. Subsonic Mode I propagation has been discussed by Walton (1990) and Herrmann and Walton (1994).

Exponential decay does not necessarily occur for all transonic crack speeds: it is necessary for the crack to extend faster than the speed of lowfrequency longitudinal waves. This observation could have some implications for the ability (or otherwise) of a viscoelastic medium to suppress transonic propagation. A more general study is in progress.

\section{Formulation of the problem}

This work is concerned with an infinite, homogeneous, isotropic viscoelastic medium whose constitutive equation is given in the form

$$
\begin{aligned}
\sigma_{i j} & =L *\left\{\lambda \delta_{i j} e_{k k}+2 \mu e_{i j}\right\} \\
& \equiv g *\left\{\lambda \delta_{i j} d e_{k k}+2 \mu d e_{i j}\right\} .
\end{aligned}
$$

The function $g(t)$ is the relaxation function and $L(t)=\dot{g}(t)$ is its derivative (interpreted in the sense of generalised functions). The symbol $*$ denotes convolution with respect to time, $t$. Explicit formulae will be developed in the case of a standard linear solid, for which the functions $g$ and $L$ are

$$
\begin{aligned}
& g(t)=\frac{1}{1+f}\left[1+f \exp \left(-\frac{1+f}{\tau} t\right)\right] H(t), \\
& L(t)=\delta(t)-\frac{f}{\tau} \exp \left(-\frac{1+f}{\tau} t\right) H(t),
\end{aligned}
$$

where $H(t)$ is the Heaviside step function and $\delta(t)$ is the Dirac delta. The parameter $f$ is positive; the ratio of the long-term (or low frequency) elastic response to the instantaneous (or high frequency) response is $1 /(1+f)$. The relaxation time so defined is $\tau /(1+f)$. It is recognised that the choice of a single relaxation function, applicable to both dilatation and shear deformation, limits the direct utility of the results; however, as mentioned in Section 1, the results obtained from this idealised problem are intended to be illustrative, and the choice of a simple relaxation function has the virtue of limiting the number of parameters. It is remarked, however, that the methodology to be developed is applicable to more general viscoelastic response, with frequency-dependent Poisson's ratio (see Appendix A).

The medium contains a semi-infinite crack which occupies, at time $t$,

$\mathbf{C}(t)=\left\{-\infty<x_{1}<v t, x_{2}= \pm 0\right\}$.

In the general case, the medium is subjected to plane-strain loading that would induce, in the absence of the crack, the stress field $\sigma_{i j}^{0}(\mathbf{x}, t)$. The presence of the crack introduces additional displacement, strain and stress fields $u_{i}(\mathbf{x}, t), e_{i j}(\mathbf{x}, t)$ and $\sigma_{i j}(\mathbf{x}, t)$. Since the response of the medium is linear, the additional stress and strain fields are related by (2.1). The additional fields also obey the equation of motion

$\sigma_{i j, j}=\rho \ddot{u}_{i}$, 
where $\rho$ is the mass density, together with a "radiation condition" that waves propagate away from the crack, and boundary conditions, that

$\sigma_{i 2}=-\sigma_{i 2}^{0}$ on $\mathbf{C}(t)$,

reflecting the fact that the crack faces must be free of traction.

In detail, however, the boundary conditions will be specialised to the form

$\sigma_{i 2}\left(x_{1}, \pm 0, t\right)=-\delta_{i 1} \delta\left(x_{1}\right) H(t) \quad$ on $\mathbf{C}(t)$.

These correspond to loading, at $x_{1}=0$, by a pair of concentrated shear forces, from time $t=0$. To the extent that all fields are zero for times $t<0$, the crack could equally well be regarded as having been stationary for $t<0$, and moving with uniform speed $v$ for $t>0$. The boundary conditions (2.6) define a "fundamental solution", from which more general loadings can be constructed by superposition. The problem thus generalises to viscoelasticity a problem recently considered for the transonic motion of a crack in an elastic medium by Huang and Gao (2001), following the precedent set by Freund (1972) in the first of his famous studies of subsonic crack growth in an elastic medium.

The advantage of expressing the constitutive relation in terms of the function $L(t)$ is that Laplace transformation yields

$\hat{\sigma}_{i j}\left(\mathbf{x}, s^{\prime}\right)=\hat{L}\left(s^{\prime}\right)\left\{\lambda \delta_{i j} \hat{e}_{k k}\left(\mathbf{x}, s^{\prime}\right)+2 \mu \hat{e}_{i j}\left(\mathbf{x}, s^{\prime}\right)\right\}$.

The hat symbol denotes the Laplace transform so that, for instance,

$\hat{L}\left(s^{\prime}\right)=\int L(t) \mathrm{e}^{-s^{\prime} t} \mathrm{~d} t=\frac{1+s^{\prime} \tau}{1+f+s^{\prime} \tau}$,

the second, explicit, form applying to the function $L(t)$ given in (2.2) for the standard linear solid.

The problem may now be formulated in terms of one for a dislocation on the plane $x_{2}=0$ or, equivalently, as a problem for the half-space $x_{2}>0$, since by symmetry the displacement components $u_{1}, u_{2}$ will be, respectively, odd and even functions of $x_{2}$. Employing the latter interpretation, the displacement component $u_{1}$ as $x_{2} \rightarrow+0$ is related to the stress component $\sigma_{12}$ so that

$u_{1}=-G_{11} * \sigma_{12}$, where $\sigma_{12}$ is evaluated as $x_{2} \rightarrow+0$ (in fact it is continuous), $G_{11}$ is the relevant component of the half-space Green's function, with "source" and "receiver" on the surface $x_{2}=0$, and $*$ here represents convolution over $x_{1}$ and $t$. The Laplace transform of $G_{11}$ (with respect to $x_{1}$ and $t$ ) takes exactly the same form as for elasticity, except that the elastic constants, and hence also wave speeds, become functions of $s^{\prime}$. Thus, denoting this double transform with a tilde,

$$
\begin{aligned}
\tilde{G}_{11}\left(k, s^{\prime}\right) & \equiv \int \mathrm{d} x_{1} \int \mathrm{d} t \mathrm{e}^{-\left(k x_{1}+s^{\prime} t\right)} G_{11}\left(x_{1}, t\right) \\
& =\frac{s^{\prime 2} \beta^{\prime}}{\rho b^{4} D^{\prime}\left(k, s^{\prime}\right)},
\end{aligned}
$$

where

$D^{\prime}\left(k, s^{\prime}\right)=\left\{\left(\beta^{\prime 2}-k^{2}\right)^{2}+4 k^{2} \alpha^{\prime} \beta^{\prime}\right\}$,

$\alpha^{\prime}=\left(s^{\prime 2} / a^{2}-k^{2}\right)^{1 / 2}, \quad \beta^{\prime}=\left(s^{\prime 2} / b^{2}-k^{2}\right)^{1 / 2}$

and the "wave speeds" $a$ and $b$ are

$a\left(s^{\prime}\right)=\left[\hat{L}\left(s^{\prime}\right)\right]^{1 / 2} c_{l}, \quad b\left(s^{\prime}\right)=\left[\hat{L}\left(s^{\prime}\right)\right]^{1 / 2} c_{s}$,

with

$c_{l}=\sqrt{(\lambda+2 \mu) / \rho}, \quad c_{s}=\sqrt{\mu / \rho}$.

The Laplace transformed version of (2.9) is

$\tilde{u}_{1}\left(k, s^{\prime}\right)=-\tilde{G}_{11}\left(k, s^{\prime}\right) \tilde{\sigma}_{12}\left(k, s^{\prime}\right)$.

For the problem of concern, it is desirable to employ a coordinate system that moves with the crack. Thus, we introduce

$\xi=x_{1}-v t$

and define

$u(\xi, t)=u_{1}\left(x_{1}, t\right), \quad \sigma(\xi, t)=\sigma_{12}\left(x_{1}, t\right)$,

with $\xi$ and $x_{1}$ related by (2.16). It is elementary to derive (Willis, 1997, 2000) that, if

$F(\xi, t)=f\left(x_{1}, t\right) \equiv f(\xi+v t, t)$ and

$G(\xi, t)=g\left(x_{1}, t\right) \equiv g(\xi+v t, t)$,

then

$F * G(\xi, t)=f * g\left(x_{1}, t\right)$,

the convolutions being taken over their natural arguments. It follows also from elementary change 
of variables that the Laplace transform $\tilde{F}(k, s)$ of $F$ is given by

$$
\begin{gathered}
\tilde{F}(k, s) \equiv \int \mathrm{d} \xi \int \mathrm{d} t \mathrm{e}^{-(k \xi+s t)} F(\xi, t)=\tilde{f}\left(k, s^{\prime}\right) ; \\
s^{\prime}=s-v k .
\end{gathered}
$$

Hence, finally, defining the transform

$\bar{F}(\zeta, s)=\tilde{F}(s \zeta, s) \equiv \tilde{f}(s \zeta, s(1-v \zeta))$,

the relation (2.15) implies

$\bar{u}(\zeta, s)=-\bar{G}(\zeta, s) \bar{\sigma}(\zeta, s)$,

where $G(\xi, t)=G_{11}(\xi+v t, t)$ and, correspondingly,

$\bar{G}(\zeta, s)=\tilde{G}_{11}(s \zeta, s(1-v \zeta))$.

Going through the transformations explicitly, the influence of viscoelasticity enters through

$\hat{L}(s(1-v \zeta))=\frac{\zeta-\zeta_{0}}{\zeta-\zeta_{1}}$,

where

$\zeta_{0}=\frac{\tau+1 / s}{v \tau}, \quad \zeta_{1}=\frac{\tau+(1+f) / s}{v \tau}$.

Correspondingly,

$\alpha^{\prime}\left(s^{\prime}\right)=s \alpha(\zeta, s), \quad \beta^{\prime}\left(s^{\prime}\right)=s \beta(\zeta, s)$,

where

$$
\begin{aligned}
& \alpha^{2}(\zeta, s)=\frac{(1-v \zeta)^{2}\left(\zeta-\zeta_{1}\right)}{c_{l}^{2}\left(\zeta-\zeta_{0}\right)}-\zeta^{2}, \\
& \beta^{2}(\zeta, s)=\frac{(1-v \zeta)^{2}\left(\zeta-\zeta_{1}\right)}{c_{s}^{2}\left(\zeta-\zeta_{0}\right)}-\zeta^{2},
\end{aligned}
$$

and

$\bar{G}(\zeta, s)=\frac{(1-v \zeta)^{2} \beta(\zeta, s)\left(\zeta-\zeta_{1}\right)^{2}}{\rho c_{s}^{4} s\left(\zeta-\zeta_{0}\right)^{2} D(\zeta, s)}$,

with

$D(\zeta, s)=\left(\beta^{2}-\zeta^{2}\right)^{2}+4 \zeta^{2} \alpha \beta$.

\subsection{The branch points of the functions $\alpha, \beta$}

In the definition of the transform $\bar{G}$ of the Green's function, it is necessary to define branches of the functions $\alpha, \beta$ so that $\operatorname{Re}(\alpha(\zeta, s))$ and $\operatorname{Re}(\beta(\zeta, s))$ are positive when $\zeta$ is pure imaginary and $s$ is real and positive. The first task is to identify the branch points. From its definition (2.25) (first term), the function $\alpha^{2}$ can be expressed

$\alpha^{2}(\zeta, s)=\frac{\left(v / c_{l}\right)^{2}-1}{\zeta-\zeta_{0}}\left(\zeta^{3}+p \zeta^{2}+q \zeta+r\right)$,

where

$p=\frac{c_{l}^{2} \zeta_{0}-\zeta_{1} v^{2}-2 v}{v^{2}-c_{l}^{2}}, \quad q=\frac{1+2 \zeta_{1} v}{v^{2}-c_{l}^{2}}$

$r=-\frac{\zeta_{1}}{v^{2}-c_{l}^{2}}$.

The function $\alpha(\zeta, s)$ thus has branch points in the $\zeta$-plane: $\zeta=\zeta_{0}>0$, and $\zeta=a_{0}, a_{1}, a_{2}$, where

$$
\begin{gathered}
\left(\zeta-a_{0}\right)\left(\zeta-a_{1}\right)\left(\zeta-a_{2}\right) \\
\equiv \zeta^{3}+p \zeta^{2}+q \zeta+r .
\end{gathered}
$$

Suppose first that $v^{2}<c_{l}^{2}$. Then $a_{0} a_{1} a_{2}=-r<0$. Therefore, at least one root is real and negative: say $a_{0}<0$, and $a_{1} a_{2}>0$. Also, $a_{0}\left(a_{1}+a_{2}\right)=$ $q-a_{1} a_{2}<0$, so $a_{1}+a_{2}>0$. Thus, both $a_{1}$ and $a_{2}$ have positive real parts. Branch cuts can therefore be chosen so that $a_{1}$ and $a_{2}$ are joined, while $\zeta_{0}$ and $a_{0}$ are joined by a cut that avoids crossing the imaginary axis by passing through infinity (Fig. 1a).

Now suppose that $v^{2}>c_{l}^{2}$ (Fig. 1b). This case is uninteresting in itself but the reasoning will apply also to the function $\beta$, with $c_{s}$ replacing $c_{l}$. It follows, since $r<0$, that there is a real root, $a_{0}>0$, and $a_{1} a_{2}>0$. This time, $q>0$ and no simple deduction can be made about $\left(a_{1}+a_{2}\right)$. It can only

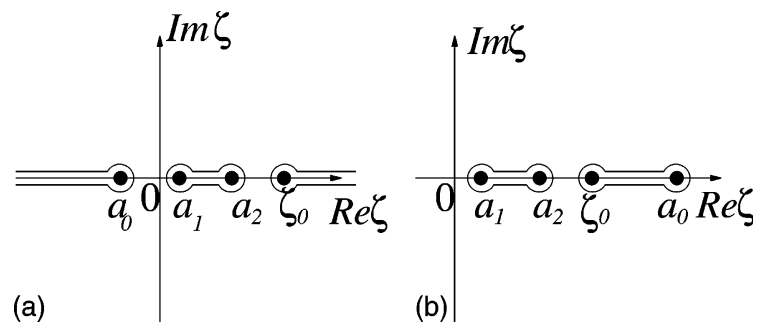

Fig. 1. The branch cuts for the function $\alpha(\zeta, s)$, for $v<c_{l}$ (a) and $v>c_{l}$ (b). 
be said that the real parts of $a_{1}$ and $a_{2}$ have the same sign. Furthermore, they remain in the same half-plane for all values of $s$. If they did not, then they would be pure imaginary (or exactly zero) for some $s$, and this would give $a_{1}+a_{2}=0$. This would imply that $a_{1} a_{2}=q$, and it would follow that $p q=r$. Inspection of this last proposed relation shows that it can never be true for any real $s>0$, when $v^{2}>c_{l}^{2}$. Branch cuts may be chosen that join $a_{1}$ and $a_{2}$, and $\zeta_{0}$ and $a_{0}$. Asymptotic analysis of the limit $s \tau \rightarrow 0$ shows, in fact, that all branch points reside in the half-plane $\operatorname{Re}(\zeta)>0$, and that one of the roots, which it is legitimate to call $a_{0}$, tends to $+\infty$ as $s \tau \rightarrow 0$. This latter fact is important because it shows that $a_{0}>\zeta_{0}$, at least for small $s \tau$. The inequality must remain true for all $s \tau$ since, if it did not, there would be some value of $s \tau$ for which $a_{0}=\zeta_{0}$. It is easy to verify by substitution that this can never happen. The inequality $a_{0}>\zeta_{0}$ ensures that $\operatorname{Re}(\alpha)>0$ for all $\zeta \in L$.

Similar reasoning applies to the function $\beta(\zeta, s)$, except that $c_{l}$ is replaced by $c_{s}$. The roots are called $b_{0}, b_{1}, b_{2}$ and the convention is adopted that $b_{0}$ is the root that is guaranteed to be real, like $a_{0}$.

\subsection{Rayleigh poles}

The function $\bar{G}(\zeta, s)$ has poles wherever $D(\zeta, s)=0$. The function $D(\zeta, s)$ is numerically equal, apart from a factor, to the function $D^{\prime}\left(k, s^{\prime}\right)$ in the numerator of (2.10), with the correspondences $s^{\prime}=s(1-v \zeta)$ and $k=s \zeta$. Now $D^{\prime}\left(k, s^{\prime}\right)=0$ when

$\frac{s^{\prime 2}}{\hat{L}\left(s^{\prime}\right)}=k^{2} c_{\mathrm{R}}^{2}$

where $c_{\mathrm{R}}$ is the speed of Rayleigh waves in an elastic medium with wave speeds $c_{l}, c_{s}$. Correspondingly, $D(\zeta, s)=0$ when

$\gamma_{\mathrm{R}}^{2}(\zeta, s) \equiv \frac{(1-v \zeta)^{2}\left(\zeta-\zeta_{1}\right)}{c_{\mathrm{R}}^{2}\left(\zeta-\zeta_{0}\right)}-\zeta^{2}=0$

(compare with the definitions (2.25) of $\alpha^{2}$ and $\beta^{2}$ ). Thus, $D(\zeta, s)=0$ when $\zeta=c_{0}, c_{1}, c_{2}$, which follow the same pattern as $a_{0}, a_{1}, a_{2}$ except that $c_{\mathrm{R}}$ replaces $c_{l}$.

\section{Riemann-Hilbert problem}

\subsection{Statement of the problem}

The relation (2.20) between $\bar{u}$ and $\bar{\sigma}$ provides a formulation of the given problem as a RiemannHilbert problem. First, the transform variable $s$ is regarded as real and positive; dependence on other, complex, values of $s$ can be established by analytic continuation, later. Dependence on the variable $\zeta$, on the other hand, will be analytic in some neighbourhood of the imaginary $\zeta$-axis (for real positive $s$ ), since two-sided Laplace transforms with respect to $\xi$ are involved.

The function $\bar{u}(\zeta, s)$, regarded as a function of $\zeta$, is, from its definition, analytic in the half-plane $\operatorname{Re}(\zeta)<0$, since the displacement component $u_{1}$ is zero ahead of the crack, so that $u(\xi, t)=0$ for all $\xi>0$. With the convention that the region to the left of the Bromwich contour $L$ for inversion of the transform (in the present case, the imaginary axis or a contour arbitrarily close to it) is considered as the "+" region, denoted $\mathscr{D}^{+}$, it is helpful to introduce the notation

$F^{+}(\zeta, s)=s^{2} \zeta \bar{u}(\zeta, s)$

(so that $F^{+}$is related to the Laplace transform of $\partial^{2} u / \partial \xi \partial t$ ). Next, the transform $\bar{\sigma}$ is given in the form

$s \bar{\sigma}(\zeta, s)=F^{-}(\zeta, s)+\phi^{+}(\zeta, s)$,

where

$$
\begin{aligned}
& \phi^{+}(\zeta, s)=s \int_{0}^{\infty} \mathrm{d} t \int_{-\infty}^{0} \mathrm{~d} \xi \mathrm{e}^{-s(\zeta \xi+t)} \sigma(\xi, t)=\frac{1}{(v \zeta-1)}, \\
& F^{-}(\zeta, s)=s \int_{0}^{\infty} \mathrm{d} t \int_{0}^{\infty} \mathrm{d} \xi \mathrm{e}^{-s(\zeta \xi+t)} \sigma(\xi, t) .
\end{aligned}
$$

The explicit form of $\phi^{+}$follows from use of the boundary condition (2.6). The function $F^{-}$is analytic in the variable $\zeta$ in the "-" region, denoted $\mathscr{D}^{-}$, to the right of $L$. It follows now from (2.20) that

$F^{+}(\zeta, s)=K(\zeta, s)\left\{F^{-}(\zeta, s)+\frac{1}{(v \zeta-1)}\right\} ; \quad \zeta \in L$, 
where

$$
\begin{aligned}
K(\zeta, s) & =-s \zeta \bar{G}(\zeta, s) \\
& =-\frac{\zeta(1-v \zeta)^{2} \beta(\zeta, s)\left(\zeta-\zeta_{1}\right)^{2}}{\rho c_{s}^{4}\left(\zeta-\zeta_{0}\right)^{2} D(\zeta, s)} .
\end{aligned}
$$

In (3.4), the structure of the solution is anticipated by taking $L$ to be the contour

$L=\{\zeta=-\delta+i y,-\infty<y<\infty\}$,

where $0<\delta \ll 1$. The relation (3.4) presents the following Riemann-Hilbert problem (Noble, 1988; Gakhov, 1966):

Find the two functions $F^{+}(\zeta, s), F^{-}(\zeta, s)$, analytic with respect to $\zeta$ in $\mathscr{D}^{+}, \mathscr{D}^{-}$respectively, which satisfy the relation (3.4) everywhere on $L$ except for the point of discontinuity of the function $K(\zeta, s)$ at infinity. The functions $F^{+}(\zeta, s), F^{-}(\zeta, s)$ admit a Hölder extension to $L$ apart perhaps from infinity, where they have to be $L^{p}$-integrable, for some $p>1$.

The solution of the Riemann-Hilbert problem (3.4) takes different forms, depending on the speed $v$ of the crack, relative to the wave speeds of the material.

\subsection{Subsonic case}

First, we study the behaviour of the function $K(\zeta, s)$ at infinity. To do this we analyse the asymptotics of the functions $\alpha$ and $\beta$. Since $v<c_{s}$, both $\alpha$ and $\beta$ behave similarly. An easy calculation, taking account of the branch cuts, shows that, as $\zeta \rightarrow-\delta \pm \mathrm{i} \infty$

$\alpha \sim \eta_{l}|\zeta|, \quad \beta \sim \eta_{s}|\zeta|$,

where

$\eta_{l}=\left|1-v^{2} / c_{l}^{2}\right|^{1 / 2}, \quad \eta_{s}=\left|1-v^{2} / c_{s}^{2}\right|^{1 / 2}$.

(modulus signs are included so that the definition will serve for all $v$.) It follows that

$$
\begin{aligned}
& K(\zeta, s)=\mathrm{i} \kappa \operatorname{sgn}\{\operatorname{Im}(\zeta)\}+O(1), \\
& \zeta \rightarrow-\delta \pm \mathrm{i} \infty,
\end{aligned}
$$

where

$\kappa=\frac{v^{2} \eta_{s}}{\rho c_{s}^{4} R(v)}$, with

$R(v)=\left(1+\eta_{s}^{2}\right)^{2}-4 \eta_{l} \eta_{s}$.

The equation $R(v)=0$ defines the speed $c_{\mathrm{R}}$ of Rayleigh waves, as introduced above.

The function $K(\zeta, s)$ is now expressed in the form (see for example, Nuller, 1976)

$K(\zeta, s)=\kappa \tan \pi c_{s} \zeta K_{0}(\zeta, s)$.

Then, $K_{0}(\zeta, s)=1+O\left(\zeta^{-1}\right)$ as $\zeta \rightarrow-\delta \pm i \infty$ and, furthermore, $K_{0}(\zeta, s)$ is non-zero at $\zeta=0$. Now since

$\tan \pi c_{s} \zeta=\frac{T^{+}(\zeta)}{T^{-}(\zeta)}$,

where

$T^{+}(\zeta)=-\frac{\Gamma\left(\frac{1}{2}-c_{s} \zeta\right)}{\Gamma\left(-c_{s} \zeta\right)}$,
$T^{-}(\zeta)=\frac{\Gamma\left(1+c_{s} \zeta\right)}{\Gamma\left(\frac{1}{2}+c_{s} \zeta\right)}$,

the Riemann-Hilbert problem (3.4) can be expressed in the form

$$
\begin{aligned}
& \frac{F^{+}(\zeta, s)}{T^{+}(\zeta)}=\kappa K_{0}(\zeta, s)\left\{\frac{F^{-}(\zeta, s)}{T^{-}(\zeta)}+\frac{1}{T^{-}(\zeta)(v \zeta-1)}\right\}, \\
& \quad \zeta \in L .
\end{aligned}
$$

Fig. 2 shows a plot (the solid curve) of $\left\{K_{0}(-\delta+\right.$ $\mathrm{i} y, s):-\infty<y<\infty\}$, for the parameter values $f=1, \tau=1, s=0.1, \delta=0.01$ and $v=0.5 c_{s}$, with Poisson's ratio $\lambda / 2(\lambda+\mu)$ for the medium taking the value $0.3 .^{2}$ The corresponding Rayleigh speed $c_{\mathrm{R}}$ is approximately $0.93 c_{s}$. The contour does not enclose the origin. Therefore, the argument of $K_{0}(\zeta, s)$ suffers no change as $\zeta$ traces the contour $L$ from $-\delta-\mathrm{i} \infty$ to $-\delta+\mathrm{i} \infty \quad(0<\delta \ll 1)$. The same behaviour has been confirmed numerically for different sets of the parameters of the problem, so long as $0 \leqslant v<c_{\mathrm{R}}$. This means that the winding number of the function $K_{0}$ is equal to zero. Therefore, the Riemann-Hilbert problem (3.15)

\footnotetext{
${ }^{2}$ This means, in effect, that time is measured in units of $\tau$, and $s$ is measured in units of $1 / \tau$. This interpretation will be adopted without comment in the sequel.
} 


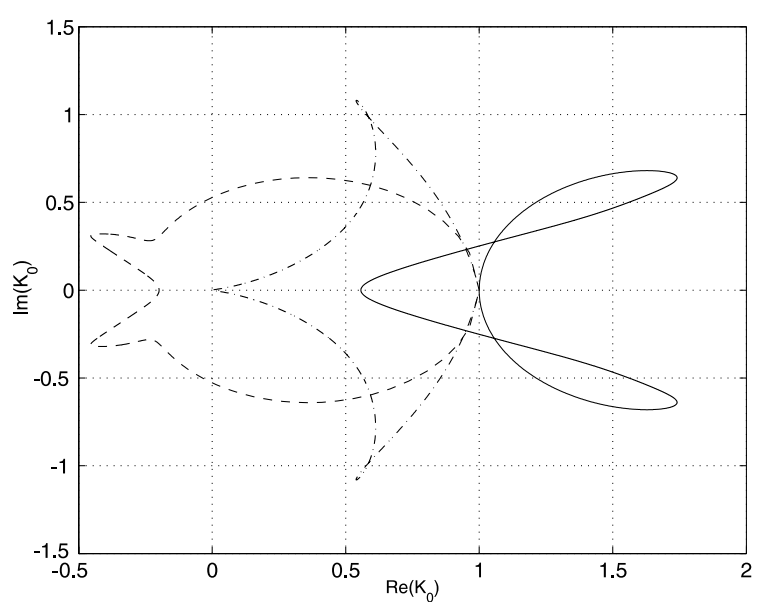

Fig. 2. The set $\left\{K_{0}(-\delta+\mathrm{i} y):-\infty<y<\infty\right\}$, for $f=1, \tau=1$, $s=0.1, \delta=0.01$ in the sub-Rayleigh case $\left(v=0.5 c_{s}\right)$ : the solid curve, in the super-Rayleigh case $\left(v=0.95 c_{s}\right)$ : the dashed curve, and in the transonic case $\left(v=1.5 c_{s}\right)$ : the dash-dotted curve.

has index zero (Gakhov, 1966) and is solvable uniquely.

If, however, $c_{\mathrm{R}}<v<c_{s}$, the argument of $K_{0}$ changes by $2 \pi$ as $L$ is traversed, as illustrated in Fig. 2 for $v=0.95 c_{s}$ (the dashed curve). The problem then has index 1 and its solution is not unique. The same situation arises in the purely elastic case. The reason for the change of index is the movement of the "Rayleigh pole" $\zeta=c_{0}$ from $\mathscr{D}^{+}$to $\mathscr{D}^{-}$as $v$ surpasses $c_{\mathrm{R}}$. The super-Rayleigh régime is not discussed further.

Now, reverting to the sub-Rayleigh régime, introduce the Cauchy integral

$$
\begin{aligned}
& \log X_{0}(\zeta, s)=\frac{1}{2 \pi i} \int_{L} \frac{\log K_{0}(\eta, s)}{\eta-\zeta} \mathrm{d} \eta \\
& \quad \arg K_{0}(\eta, s) \in(-\pi, \pi)
\end{aligned}
$$

Let the limit values of the function $X_{0}(\zeta, s)$, as $\zeta$ approaches $\eta \in L$ from $\mathscr{D}^{+}$and $\mathscr{D}^{-}$, be $X_{0}^{+}(\eta, s)$ and $X_{0}^{-}(\eta, s)$, respectively. Then by the SokhotskiPlemelj formulae,

$$
K_{0}(\eta, s)=\frac{X_{0}^{+}(\eta, s)}{X_{0}^{-}(\eta, s)}, \quad \eta \in L
$$

The function $K(\eta, s)$ admits the factorisation

$K(\eta, s)=\frac{X^{+}(\eta, s)}{X^{-}(\eta, s)}, \quad \eta \in L$,

where

$X^{+}(\eta, s)=\kappa T^{+}(\eta) X_{0}^{+}(\eta, s)$,

$X^{-}(\eta, s)=T^{-}(\eta) X_{0}^{-}(\eta, s)$.

The Riemann-Hilbert problem (3.15) can now be expressed

$$
\begin{aligned}
& \frac{F^{+}(\eta, s)}{X^{+}(\eta, s)}-\frac{1}{X^{-}(1 / v, s)(v \eta-1)} \\
& \quad=\frac{F^{-}(\eta, s)}{X^{-}(\eta, s)}+\left(\frac{1}{X^{-}(\eta, s)}-\frac{1}{X^{-}(1 / v, s)}\right) \frac{1}{(v \eta-1)},
\end{aligned}
$$

$\eta \in L$.

The function on the left side of (3.20) is analytic in $\mathscr{D}^{+}$, while that on the right side is analytic in $\mathscr{D}^{-}$. Therefore, together, they define an entire function. This function tends to zero as its argument tends to infinity, and hence, by Liouville's theorem, is equal to zero everywhere in the $\zeta$-plane. Therefore, the solution of the Riemann-Hilbert problem becomes

$$
\begin{aligned}
& F^{+}(\zeta, s)=\frac{X^{+}(\zeta, s)}{X^{-}(1 / v, s)(v \zeta-1)}, \quad \zeta \in \mathscr{D}^{+}, \quad \\
& F^{-}(\zeta, s)=\left[\frac{X^{-}(\zeta, s)}{X^{-}(1 / v, s)}-1\right] \frac{1}{(v \zeta-1)}, \quad \zeta \in \mathscr{D}^{-} .
\end{aligned}
$$

\subsection{Transonic case}

In the transonic régime, the properties of the branch $\alpha(\zeta, s)$ are the same as in the subsonic case. However, the function $\beta(\zeta, s)$ is not discontinuous at infinity as it was in the subsonic case, because the cuts joining the branch points all lie in $\mathscr{D}^{-}$. In fact, $\beta \sim-\eta_{s} \zeta, \zeta \rightarrow-\delta \pm \mathrm{i} \infty$, while the formula (3.7) (first term) for the asymptotic behaviour of $\alpha(\zeta, s)$ remains valid. The coefficient $K(\zeta, s)$ of the Riemann-Hilbert problem is discontinuous at infinity:

$$
\begin{gathered}
K(\zeta, s)=\frac{v^{2} \eta_{s}}{\rho c_{s}^{4}}\left[\left(\frac{v^{2}}{c_{s}^{2}}-2\right)^{2} \pm 4 \mathrm{i} \eta_{s} \eta_{l}\right]^{-1}+O\left(\frac{1}{\zeta}\right), \\
\zeta \rightarrow-\delta \pm \mathrm{i} \infty
\end{gathered}
$$


We now split the function $K(\zeta, s)$ into the following factors

$K(\zeta, s)=\frac{\kappa}{\cos \pi \kappa_{0}\left(\tan \pi c_{s} \zeta+\tan \pi \kappa_{0}\right)} K_{0}(\zeta, s)$,

where

$\kappa=\frac{v^{2} \cos \pi \kappa_{0}}{4 \rho c_{s}^{4} \eta_{l}}$,

$\kappa_{0}=\frac{1}{\pi} \tan ^{-1} \frac{\left(v^{2} / c_{s}^{2}-2\right)^{2}}{4 \eta_{s} \eta_{l}} \in\left[0, \frac{1}{2}\right)$.

The advantage of the representation (3.24) is first, in the relatively simple factorisation of the trigonometric function (see for example, Antipov and Arutyunyan, 1991):

$\cos \pi \kappa_{0}\left(\tan \pi c_{s} \zeta+\tan \pi \kappa_{0}\right)=\frac{T^{-}(\zeta)}{T^{+}(\zeta)}$,

where

$T^{+}(\zeta)=\frac{\Gamma\left(1-\kappa_{0}-c_{s} \zeta\right)}{\Gamma\left(\frac{1}{2}-c_{s} \zeta\right)}$,

$T^{-}(\zeta)=\frac{\Gamma\left(\frac{1}{2}+c_{s} \zeta\right)}{\Gamma\left(\kappa_{0}+c_{s} \zeta\right)}$,

and second, in the continuity of the function $K_{0}(\zeta, s)$ at infinity. Indeed,

$$
\begin{aligned}
K_{0}(\zeta, s) & =\kappa^{-1} \cos \pi \kappa_{0}\left(\tan \pi c_{s} \zeta+\tan \pi \kappa_{0}\right) K(\zeta, s) \\
& =1+O\left(\zeta^{-1}\right), \quad \zeta \rightarrow-\delta \pm \mathrm{i} \infty .
\end{aligned}
$$

We have confirmed numerically, for different values of the parameters of the problem, that $K_{0}(\zeta, s)$ has index zero (see Fig. 2 for $v=1.5 c_{s}$ : the dashdotted curve). Therefore, the factorisation of the function $K_{0}(\zeta, s)$ is constructed as in the subsonic case and it is given by formulae (3.16) and (3.17). Analysis of the behaviour of the functions $X_{0}^{ \pm}(\zeta, s)$ and $T^{ \pm}(\zeta)$ at infinity shows that

$$
\begin{aligned}
& X_{0}^{ \pm}(\zeta, s) \sim 1, \quad T^{ \pm}(\zeta) \sim\left(\mp c_{s} \zeta\right)^{1 / 2-\kappa_{0}}, \\
& \quad \zeta \rightarrow \infty, \quad \zeta \in \mathscr{D}^{ \pm} .
\end{aligned}
$$

Therefore, the uniqueness of the solution of the Riemann-Hilbert problem (3.4) is guaranteed in the class of functions which possess the following asymptotics at infinity
$F^{ \pm}(\zeta, s)=O\left(\zeta^{-\kappa_{0}-1 / 2}\right), \quad \zeta \rightarrow \infty, \quad \zeta \in \mathscr{D}^{ \pm}$.

The functions $F^{ \pm}(\zeta, s)$ are determined by formulae (3.21) and (3.22), where $T^{ \pm}(\zeta), \kappa$ and $K_{0}(\zeta, s)$ have to be changed so that they conform to (3.27), (3.25), (3.24), respectively. The form of the singularity of the traction $\hat{\sigma}(\xi, s)$ at the crack tip $\xi=0$ follows directly from the Tauberian theorem:

$\hat{\sigma}(\xi, s)=O\left(\xi^{\kappa_{0}-1 / 2}\right), \quad \xi \rightarrow 0$.

Formula (3.25) for $\kappa_{0}$ indicates that this parameter is independent of the viscoelastic parameters $\tau$ and $f$, and $\kappa_{0}=0$ when $v$ coincides with Eshelby's special speed $\sqrt{2} c_{s}$, which he derived for the elastic case.

\section{Stress singularity coefficients}

\subsection{Stress intensity factor: sub-Rayleigh case}

We start with the sub-Rayleigh régime, for which $\kappa_{0}=0$. The singularity takes its usual square-root form and it is appropriate to measure its strength via the stress intensity factor $K_{\mathrm{II}}$. Its Laplace transform is given as follows:

$\hat{K}_{\mathrm{II}}(s)=\lim _{\xi \rightarrow+0} \sqrt{2 \pi \xi} \hat{\sigma}(\xi, s)$.

Then by the Abelian theorem, from (3.3),

$F^{-}(\zeta, s) \sim \frac{s^{1 / 2} \hat{K}_{\mathrm{II}}(s)}{\sqrt{2}} \zeta^{-1 / 2}, \quad \zeta \rightarrow \infty, \quad \zeta \in \mathscr{D}^{-}$.

On the other hand, directly from (3.21), (3.22) we establish

$F^{-}(\zeta, s) \sim \frac{\sqrt{c_{s}}}{v X^{-}(1 / v, s)} \zeta^{-1 / 2}, \quad \zeta \rightarrow \infty, \quad \zeta \in \mathscr{D}^{-}$.

Comparing the above two formulae yields

$$
\begin{aligned}
\hat{K}_{\mathrm{II}}(s) & =\frac{\sqrt{2 c_{s}}}{s^{1 / 2} v X^{-}(1 / v, s)} \\
& =\frac{\sqrt{2 c_{s}} \Gamma\left(\frac{1}{2}+c_{s} / v\right)}{s^{1 / 2} v \Gamma\left(1+c_{s} / v\right) X_{0}^{-}(1 / v, s)} .
\end{aligned}
$$


A representation for $X_{0}^{-}(1 / v, s)$ suitable for computation is discussed in Appendix B.

\subsection{Singularity coefficient in the transonic case}

The singular behaviour of the stress is $\sigma(\xi, t) \sim k(t) \xi^{k_{0}-1 / 2}$ as $\xi \rightarrow+0$. Correspondingly, the Laplace transform of $k(t)$ is defined as

$\hat{k}(s)=\lim _{\xi \rightarrow+0} \xi^{1 / 2-\kappa_{0}} \hat{\sigma}(\xi, s)$.

By expanding out the function $X^{-}(\zeta, s)$ at infinity, we get

$$
\begin{aligned}
& F^{-}(\zeta, s) \sim \frac{c_{s}}{v X^{-}(1 / v, s)}\left(c_{s} \zeta\right)^{-\kappa_{0}-1 / 2}, \quad \zeta \rightarrow \infty, \\
& \quad \zeta \in \mathscr{D}^{-} .
\end{aligned}
$$

By the Tauberian theorem,

$\hat{\sigma}(\xi, s) \sim \frac{\left(s \xi / c_{s}\right)^{\kappa_{0}-1 / 2}}{v X^{-}(1 / v, s) \Gamma\left(\frac{1}{2}+\kappa_{0}\right)}, \quad \xi \rightarrow+0$

and therefore, by the definition (4.5), the coefficient $\hat{k}(s)$ becomes

$$
\begin{aligned}
\hat{k}(s) & =\frac{\left(s / c_{s}\right)^{\kappa_{0}-1 / 2}}{v X^{-}(1 / v, s) \Gamma\left(\frac{1}{2}+\kappa_{0}\right)} \\
& =\frac{\Gamma\left(\kappa_{0}+c_{s} / v\right)\left(s / c_{s}\right)^{\kappa_{0}-1 / 2}}{v \Gamma\left(\frac{1}{2}+c_{s} / v\right) \Gamma\left(\frac{1}{2}+\kappa_{0}\right) X_{0}^{-}(1 / v, s)} .
\end{aligned}
$$

The representation (B.1) is again employed for the computation of $X_{0}^{-}(1 / v, s)$, in this case with allowance for a discontinuity of the function $H(w, s)$ defined in Appendix B.

\subsection{Inverse Laplace transformation}

The formula for inversion of the Laplace transform gives, for the sub-Rayleigh case,

$K_{\mathrm{II}}(t)=\frac{1}{2 \pi \mathrm{i}} \int_{\gamma-\mathrm{i} \infty}^{\gamma+\mathrm{i} \infty} \hat{K}_{\mathrm{II}}(s) \mathrm{e}^{s t} \mathrm{~d} s, \quad \gamma>0$.

The formula for evaluation of $k(t)$ in the transonic case is similar. We next transform the formula (4.9) to a form convenient for numerical evaluation. The Laplace transform of the stress-intensity factor,

$\hat{K}_{\mathrm{II}}(s)=\int_{0}^{\infty} \mathrm{e}^{-s t} K_{\mathrm{II}}(t) \mathrm{d} t, \quad s=\gamma+\mathrm{i} \omega$, can be written as follows

$$
\begin{aligned}
\operatorname{Re}\left\{\hat{K}_{\text {II }}(\gamma+\mathrm{i} \omega)\right\}+\mathrm{i} \operatorname{Im}\left\{\hat{K}_{\mathrm{II}}(\gamma+\mathrm{i} \omega)\right\} \\
=\frac{1}{2} \int_{-\infty}^{\infty} \mathrm{e}^{-\gamma|t|} K_{\mathrm{II}}(|t|) \cos \omega t \mathrm{~d} t \\
\quad-\frac{\mathrm{i}}{2} \int_{-\infty}^{\infty} \mathrm{e}^{-\gamma|t|} \operatorname{sgn} t K_{\mathrm{II}}(|t|) \sin \omega t \mathrm{~d} t .
\end{aligned}
$$

Fourier inversion now yields, when $t>0$,

$$
\begin{aligned}
& K_{\mathrm{II}}(t)=\frac{2}{\pi} \mathrm{e}^{\gamma t} \int_{0}^{\infty} \operatorname{Re}\left\{\hat{K}_{\mathrm{II}}(\gamma+\mathrm{i} \omega)\right\} \cos \omega t \mathrm{~d} \omega, \\
& K_{\mathrm{II}}(t)=-\frac{2}{\pi} \mathrm{e}^{\gamma t} \int_{0}^{\infty} \operatorname{Im}\left\{\hat{K}_{\mathrm{II}}(\gamma+\mathrm{i} \omega)\right\} \sin \omega t \mathrm{~d} \omega
\end{aligned}
$$

(essentially this device was employed, for example, by Abate and Whitt, 1995). Looking at formulae (4.12) we see that they both define the same function $K_{\mathrm{II}}(t)$. Therefore they can be used for testing a numerical algorithm. On the other hand, since the inverse Laplace transform (4.9) is independent of $\gamma>0$, the numerical values of the integrals (4.12) have to be the same for judicious values of the parameter $\gamma$ (if $\gamma \rightarrow \infty$ then $e^{\gamma t} \rightarrow \infty$ and the error of approximation grows to infinity).

The convergence of the integrals (4.12) may be improved as follows. In the sub-Rayleigh case, letting $s \rightarrow \infty$ gives

$X^{-}\left(\frac{1}{v}, s\right) \rightarrow X_{\mathrm{e}}^{-}\left(\frac{1}{v}\right), \quad s \rightarrow \infty$,

where $X_{\mathrm{e}}^{-}(\zeta)$ is the corresponding kernel for the elastic problem. This provides the Laplace transform of the stress intensity factor $K_{\mathrm{II}, \mathrm{e}}(t)$ in the elastic case:

$\hat{K}_{\mathrm{II}, \mathrm{e}}(s)=\frac{\sqrt{2 c_{s}}}{s^{1 / 2} v X_{\mathrm{e}}^{-}(1 / v)}$,

which admits the exact Laplace inversion

$K_{\mathrm{II}, \mathrm{e}}(t)=\frac{1}{v X_{\mathrm{e}}^{-}(1 / v)} \sqrt{\frac{2 c_{s}}{\pi t}}$.

Doing the same in the transonic case, we get

$\hat{k}_{\mathrm{e}}(s)=\frac{\left(s / c_{s}\right)^{\kappa_{0}-1 / 2}}{v \Gamma\left(\kappa_{0}+1 / 2\right) X_{\mathrm{e}}^{-}(1 / v)}$. 
By using the Laplace transformation formula

$\mathscr{L}\left[t^{v}\right]=\frac{\Gamma(v+1)}{s^{v+1}}, \quad v>-1, \quad \operatorname{Re}(s)>1$,

we obtain the explicit representation for the factor $k_{\mathrm{e}}(t)$ :

$k_{\mathrm{e}}(t)=\frac{\cos \pi \kappa_{0} c_{s}^{1 / 2-\kappa_{0}}}{\pi v t^{1 / 2+\kappa_{0}} X_{\mathrm{e}}^{-}(1 / v)}$.

We notice that in the limiting case $s \rightarrow \infty$, the branch points $a_{0}, b_{0}$ and $\zeta_{0}$ coincide and cancel each other. The functions $\alpha, \beta$ in the $\zeta$-plane have the branch points $\zeta=\left(c_{l} \pm v\right)^{-1}$ and $\zeta=\left(c_{s} \pm v\right)^{-1}$, respectively.

In fact, for both sub-Rayleigh $\left(\kappa_{0}=0\right)$ and transonic régimes, the difference between the two functions $X^{-}(1 / v, s) s^{\kappa_{0}-1 / 2}$ and $X_{\mathrm{e}}^{-}(1 / v) s^{\kappa_{0}-1 / 2}$ is visible only for $0<\omega<6, \omega=\operatorname{Im}(s)$. The function

$W(\omega, \kappa)=\left[\frac{X_{\mathrm{e}}^{-}(1 / v)}{X^{-}(1 / v, \gamma+\mathrm{i} \omega)}-1\right](\gamma+\mathrm{i} \omega)^{\kappa-1 / 2}$

is plotted in Fig. 3 (the transonic case). Therefore, for numerical purposes, it is effective to represent the stress-intensity factor for the sub-Rayleigh case in the form

$K_{\mathrm{II}}(t)=\frac{\sqrt{2 c_{s}}}{v X_{\mathrm{e}}^{-}(1 / v)}\left[\frac{1}{\sqrt{\pi t}}+I_{1}(t)\right]$

where

$$
\begin{aligned}
I_{1}(t) & =\frac{2}{\pi} \mathrm{e}^{\gamma t} \int_{0}^{\infty} \operatorname{Re}\{W(\omega, 0)\} \cos \omega t \mathrm{~d} \omega \\
& =-\frac{2}{\pi} \mathrm{e}^{\gamma t} \int_{0}^{\infty} \operatorname{Im}\{W(\omega, 0)\} \sin \omega t \mathrm{~d} \omega .
\end{aligned}
$$

If the crack propagates at a transonic speed, then the factor $k(t)$ can be evaluated as follows

$k(t)=\frac{\cos \pi \kappa_{0} c_{s}^{1 / 2-\kappa_{0}}}{\pi v X_{\mathrm{e}}^{-}(1 / v)}\left[t^{-\kappa_{0}-1 / 2}+I_{2}(t)\right]$

where

$$
\begin{aligned}
I_{2}(t)= & \frac{2}{\pi} \mathrm{e}^{\gamma t} \Gamma\left(\frac{1}{2}-\kappa_{0}\right) \int_{0}^{\infty} \operatorname{Re}\left\{W\left(\omega, \kappa_{0}\right)\right\} \\
& \times \cos \omega t \mathrm{~d} \omega \\
= & -\frac{2}{\pi} \mathrm{e}^{\gamma t} \Gamma\left(\frac{1}{2}-\kappa_{0}\right) \int_{0}^{\infty} \operatorname{Im}\left\{W\left(\omega, \kappa_{0}\right)\right\} \\
& \times \sin \omega t \mathrm{~d} \omega .
\end{aligned}
$$

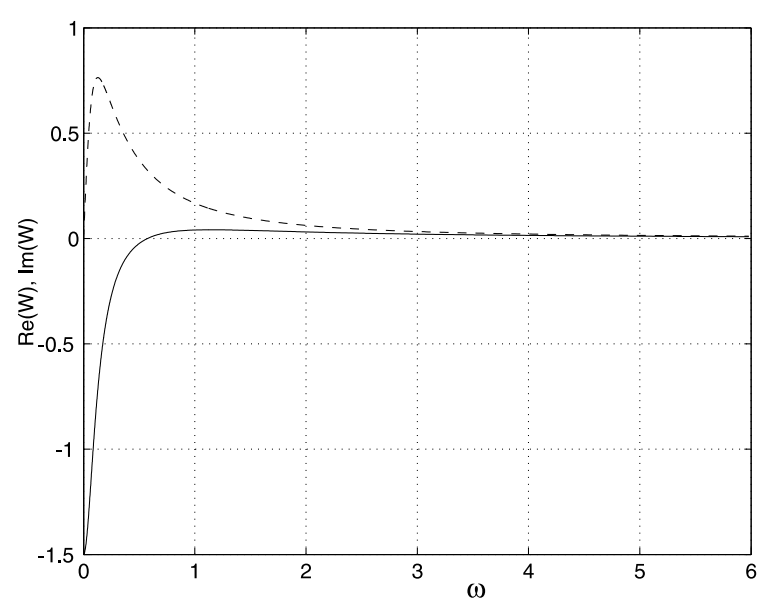

Fig. 3. The real (solid line) and imaginary (dashed line) parts of the function $W\left(\omega, \kappa_{0}\right)$ for $f=1, \tau=1, \gamma=0.1, v=1.5 c_{s}$.

\subsection{Computations}

The integrals (4.21), (4.23) have rapidly oscillating factors when $t$ is large. They were evaluated by approximating the non-oscillating factors as quadratic functions on sub-intervals, and then integrating the products of quadratic functions with sines or cosines analytically (in the absence of the sines or cosines, this reproduces Simpson's rule). The infinite range was dealt with by simple truncation. Convergence of the resulting sums was accelerated by applying Euler summation, though this was not strictly necessary, for the purpose of evaluation to the accuracy required for graphical presentation.

Fig. 4 shows $K_{\mathrm{II}}(t)$ for a sub-Rayleigh speed $\left(v=0.5 c_{s}\right)$, and Fig. 5 shows a sample plot of $k(t)$ for a transonic speed $\left(v=1.5 c_{s}\right)$ for some values of the parameters $f$ and $\tau: f=1, \tau=1$, and $f=5, \tau=3$. They are chosen such that the relaxation time $\tau /(1+f)$ is the same in both cases but give different long-time moduli. The dashdotted curves on the figures give the results for the elastic medium (obtained by letting $\tau \rightarrow \infty$ ), while the solid and dashed curves are for the viscoelastic cases. At short times the curves tend to coincide, because the strain-rate induced by the delta-function loading is infinite, initially. Later, however, as the distance of the crack tip from the point of application of the load increases, the viscoelasticity 


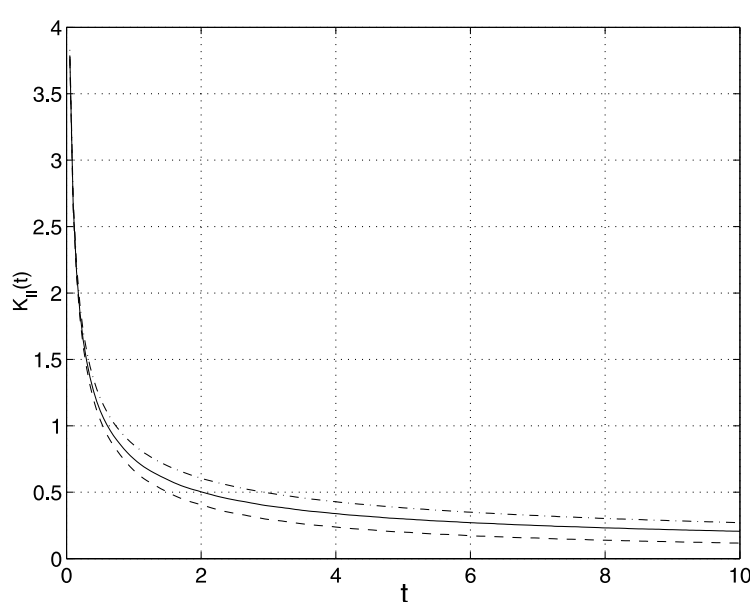

Fig. 4. The stress-intensity factor $K_{\mathrm{II}}(t)$ in the sub-Rayleigh case $v=0.5 c_{s}$ for $f=1, \tau=1$ (the solid curve), $f=5, \tau=3$ (the dashed curve) and for the elastic case (the dash-dotted curve).

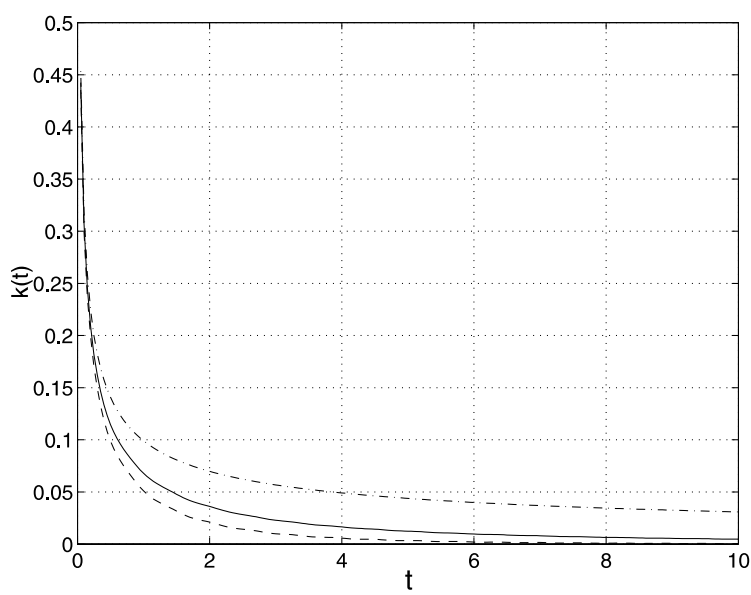

Fig. 5. The stress singularity coefficient $k(t)$ in the transonic case $v=1.5 c_{s}$ for $f=1, \tau=1$ (the solid curve), $f=5, \tau=3$ (the dashed curve) and for the elastic case (the dash-dotted curve).

progressively reduces the coefficient in comparison with its elastic value. The reductions in $K_{\mathrm{II}}(t)$ and $k(t)$ are greater in the case $f=5$, which corresponds to the more extreme reduction of the longtime moduli. This effect of viscoelastic decay would be more pronounced even after a short time, for any smooth loading: the effect of any time-independent loading, for example, is obtained by forming a convolution of the given result with the loading function.

\section{Shear traction ahead of the crack}

Inverting the transform (3.3) with respect to $\zeta$ gives

$$
\begin{aligned}
\hat{\sigma}(\xi, s) & =\frac{1}{2 \pi \mathrm{i}} \int_{L} F^{-}(\zeta, s) \mathrm{e}^{s \zeta \xi} \mathrm{d} \zeta \\
& =\frac{1}{2 \pi \mathrm{i} s} \int_{L^{\prime}} F^{-}(k / s, s) \mathrm{e}^{k \xi} \mathrm{d} k .
\end{aligned}
$$

The second form will be useful below, when the reciprocal space and time variables will need to be uncoupled. (The line $L^{\prime}$ is the image of $L$ under the transformation $k=s \zeta$.)

A convenient way to evaluate the transform is to employ the analytic continuation of $F^{-}$into $\mathscr{D}^{+}$, exploiting the basic Riemann-Hilbert relation (3.4):

$F^{-}(\zeta, s)=\frac{F^{+}(\zeta, s)}{K(\zeta, s)}-\frac{1}{(v \zeta-1)}$.

The second term reproduces the delta-function loading applied to the crack faces. Thus, when $\xi>0$,

$\hat{\sigma}(\xi, s)=\hat{\sigma}_{a}(\xi, s)+\hat{\sigma}_{b}(\xi, s)$,

where

$$
\begin{aligned}
\hat{\sigma}_{a}(\xi, s)= & \frac{-1}{2 \pi \mathrm{i}} \int_{L} \frac{4 \rho c_{s}^{4}\left(\zeta-\zeta_{0}\right)^{2} \zeta \alpha(\zeta, s) F^{+}(\zeta, s)}{(1-v \zeta)^{2}\left(\zeta-\zeta_{1}\right)^{2}} \\
& \times \mathrm{e}^{s \zeta \xi} \mathrm{d} \zeta
\end{aligned}
$$

and

$$
\begin{aligned}
\hat{\sigma}_{b}(\zeta, s)= & \frac{-1}{2 \pi \mathrm{i}} \int_{L} \frac{\rho c_{s}^{4}\left(\zeta-\zeta_{0}\right)^{2}\left(\beta^{2}-\zeta^{2}\right)^{2} F^{+}(\zeta, s)}{\zeta(1-v \zeta)^{2}\left(\zeta-\zeta_{1}\right)^{2} \beta(\zeta, s)} \\
& \times \mathrm{e}^{s \zeta \xi} \mathrm{d} \zeta
\end{aligned}
$$

having substituted the explicit form (3.5) for $K(\zeta, s)$. The only functions in the integrands that may not be analytic in $\mathscr{D}^{+}$are $\alpha$ and $\beta$. For subRayleigh $v$ (so that, a fortiori, $\left.v<c_{s}\right), \alpha(\zeta, s)$ and $\beta(\zeta, s)$ are analytic in $\mathscr{D}^{+}$, except for cuts on the segments $\left(-\infty, a_{0}\right]$ and $\left(-\infty, b_{0}\right]$ respectively. In the transonic range $c_{s}<v<c_{l}$, the function $\beta$ is analytic in $\mathscr{D}^{+}$while $\alpha$ is analytic in $\mathscr{D}^{+}$except on the cut $\left(-\infty, a_{0}\right]$. 
The contour $L$ may now be deformed into $\mathscr{D}^{+}$, by use of Jordan's lemma when $\xi>0$. This gives, for $\hat{\sigma}_{a}$,

$$
\begin{aligned}
\hat{\sigma}_{a}(\xi, s)= & \frac{-1}{\pi \mathrm{i}} \\
& \times \int_{a_{0}}^{-\infty+0 \mathrm{i}} \frac{4 \rho c_{s}^{4} \zeta\left(\zeta-\zeta_{0}\right)^{2} \alpha(\zeta, s) F^{+}(\zeta, s)}{(1-v \zeta)^{2}\left(\zeta-\zeta_{1}\right)^{2}} \\
& \times \mathrm{e}^{s \zeta \xi} \mathrm{d} \zeta .
\end{aligned}
$$

The fact that $\alpha$ simply changes sign at points on opposite sides of the branch cut was exploited here. Similarly, if $v<c_{\mathrm{R}}$,

$$
\begin{aligned}
\hat{\sigma}_{b}(\xi, s)= & \frac{-1}{\pi \mathrm{i}} \\
& \times \int_{b_{0}}^{-\infty+0 \mathrm{i}} \frac{\rho c_{s}^{4}\left(\zeta-\zeta_{0}\right)^{2}\left(\beta^{2}-\zeta^{2}\right)^{2} F^{+}(\zeta, s)}{\zeta(1-v \zeta)^{2}\left(\zeta-\zeta_{1}\right)^{2} \beta(\zeta, s)} \\
& \times \mathrm{e}^{s \zeta \xi} \mathrm{d} \zeta,
\end{aligned}
$$

whereas, if $v>c_{s}$, then $\sigma_{b}(\xi, s)=0$. If $v>c_{l}$, then also $\sigma_{a}(\xi, s)=0$.

\subsection{Asymptotic form of the traction for large time}

The representations given above permit the analytic deduction of the long-time asymptotic form of $\sigma(\xi, t)$. This corresponds to the asymptotic form of its Laplace transform, as $s \rightarrow 0$. It is important, however, that the transform variable $(k$, say) reciprocal to $\xi$ should remain fixed. This is the reason for the introduction of $k$ into the second of relations (5.1). It is helpful to introduce the definitions

$\lambda_{a}=-\lim _{s \rightarrow 0}\left(s a_{0}\right), \quad \lambda_{b}=-\lim _{s \rightarrow 0}\left(s b_{0}\right)$.

\subsubsection{Subsonic range}

Consider first the range $0 \leqslant v<c_{\mathrm{R}}$. Employ the substitution $\zeta=k / s$ in (5.6), followed by the further substitution $k=s a_{0}-k^{\prime}$. This gives

$F^{+}\left(\frac{s a_{0}-k^{\prime}}{s}, s\right) \sim \frac{\kappa c_{s}^{1 / 2}\left(\frac{k^{\prime}+\lambda_{a}}{s}\right)^{-1 / 2} \Gamma\left(\frac{1}{2}+c_{s} / v\right)}{v \Gamma\left(1+c_{s} / v\right) X_{0}^{-}(1 / v, 0)}$.

Hence, also substituting for $\kappa$ using (3.10),

$$
\begin{aligned}
\hat{\sigma}_{a}(\xi, s) \sim & -\frac{\mathrm{e}^{-\lambda_{a} \xi} c_{s}^{1 / 2} \Gamma\left(\frac{1}{2}+c_{s} / v\right) 4 \eta_{l} \eta_{s}}{\pi v R(v) \Gamma\left(1+c_{s} / v\right) X_{0}^{-}(1 / v, 0) s^{1 / 2}} \\
& \times J\left(\lambda_{a}, 0 ; \xi\right),
\end{aligned}
$$

where

$$
\begin{aligned}
J(\lambda, \kappa ; \xi)= & \int_{0}^{\infty}\left(k^{\prime}+\lambda\right)^{-1 / 2-\kappa} \\
& \times\left(\frac{k^{\prime}+\lambda+1 / v \tau}{k^{\prime}+\lambda+(1+f) / v \tau}\right)^{2} \mathrm{e}^{-k^{\prime} \xi} \mathrm{d} k^{\prime} .
\end{aligned}
$$

Applying the Tauberian theorem now gives

$$
\begin{aligned}
\sigma_{a}(\xi, t) \sim & -\frac{\mathrm{e}^{-\lambda_{a} \xi} \Gamma\left(\frac{1}{2}+c_{s} / v\right) 4 \eta_{l} \eta_{s}\left(c_{s} t\right)^{-1 / 2}}{\pi^{3 / 2}\left(v / c_{s}\right) R(v) \Gamma\left(1+c_{s} / v\right) X_{0}^{-}(1 / v, 0)} \\
& \times J\left(\lambda_{a}, 0 ; \xi\right)
\end{aligned}
$$

as $t \rightarrow \infty$.

Performing the analogous calculations for $\sigma_{b}$ gives the results

$$
\begin{aligned}
\hat{\sigma}_{b}(\xi, s) \sim & \frac{\mathrm{e}^{-\lambda_{b} \xi} c_{s}^{1 / 2} \Gamma\left(\frac{1}{2}+c_{s} / v\right)\left(1+\eta_{s}^{2}\right)^{2}}{\pi v R(v) \Gamma\left(1+c_{s} / v\right) X_{0}^{-}(1 / v, 0) s^{1 / 2}} \\
& \times J\left(\lambda_{b}, 0 ; \xi\right),
\end{aligned}
$$

and

$$
\begin{aligned}
\sigma_{b}(\xi, t) \sim & \frac{\mathrm{e}^{-\lambda_{b} \xi} \Gamma\left(\frac{1}{2}+c_{s} / v\right)\left(1+\eta_{s}^{2}\right)^{2}\left(c_{s} t\right)^{-1 / 2}}{\pi^{3 / 2}\left(v / c_{s}\right) R(v) \Gamma\left(1+c_{s} / v\right) X_{0}^{-}(1 / v, 0)} \\
& \times J\left(\lambda_{b}, 0 ; \xi\right)
\end{aligned}
$$

as $t \rightarrow \infty$.

$\hat{\sigma}_{a}(\xi, s)=\frac{\mathrm{e}^{s a_{0} \xi}}{\pi \mathrm{i}} \int_{0}^{\infty-0 \mathrm{i}} \frac{4 \rho c_{s}^{4}\left(s a_{0}-k^{\prime}\right)\left[s\left(a_{0}-\zeta_{0}\right)-k^{\prime}\right]^{2} \alpha\left(\frac{s a_{0}-k^{\prime}}{s}, s\right) F^{+}\left(\frac{s a_{0}-k^{\prime}}{s}, s\right)}{\left[s-v\left(s a_{0}-k^{\prime}\right)\right]^{2}\left[s\left(a_{0}-\zeta_{1}\right)-k^{\prime}\right]^{2}} \mathrm{e}^{-k^{\prime} \xi} \mathrm{d} k^{\prime}$.

Now letting $s \rightarrow 0$, it follows that $\alpha \sim \mathrm{i} \eta_{l}\left(k^{\prime}+\lambda_{a}\right) /$ $s$ and
The asymptotic form of the stress intensity factor as $t \rightarrow \infty$ follows directly from the formulae 
given above, by letting $\xi \rightarrow 0$. The results are

$\hat{K}_{\mathrm{II}}(s) \sim \frac{\sqrt{2} \Gamma\left(\frac{1}{2}+c_{s} / v\right)\left(c_{s} s\right)^{-1 / 2}}{\left(v / c_{s}\right) \Gamma\left(1+c_{s} / v\right) X_{0}^{-}(1 / v, 0)} \quad$ as $s \rightarrow 0$,

$K_{\mathrm{II}}(t) \sim \frac{\sqrt{2} \Gamma\left(\frac{1}{2}+c_{s} / v\right)\left(c_{s} t\right)^{-1 / 2}}{\pi^{1 / 2}\left(v / c_{s}\right) \Gamma\left(1+c_{s} / v\right) X_{0}^{-}(1 / v, 0)} \quad$ as $t \rightarrow \infty$.

It is noted that (5.16) is in agreement with (4.4).

\subsubsection{Transonic range}

Considering now the transonic range $c_{s}<v<$ $c_{l}$, the function $\sigma_{b}$ is zero, by Cauchy's theorem, because its integrand is analytic in $\mathscr{D}^{+}$. Thus, $\sigma(\xi, t) \equiv \sigma_{a}(\xi, t)$. Eq. (5.9) still applies but now, when $s \rightarrow 0$,

$$
\begin{aligned}
& F^{+}\left(\frac{s a_{0}-k^{\prime}}{s}, s\right) \\
& \quad \sim-\frac{\kappa c_{s}^{1 / 2-\kappa_{0}}\left(\frac{k^{\prime}+\lambda_{a}}{s}\right)^{-1 / 2-\kappa_{0}} \Gamma\left(\kappa_{0}+c_{s} / v\right)}{v \Gamma\left(\frac{1}{2}+c_{s} / v\right) X_{0}^{-}(1 / v, 0)},
\end{aligned}
$$

with $\kappa$ now given by (3.25). The result is, as $s \rightarrow 0$,

$$
\begin{aligned}
\hat{\sigma}(\xi, s) \sim & \frac{\mathrm{e}^{-\lambda_{a} \xi} c_{s}^{1 / 2-\kappa_{0}} \Gamma\left(\kappa_{0}+c_{s} / v\right) \cos \pi \kappa_{0} s^{\kappa_{0}-1 / 2}}{\pi v \Gamma\left(\frac{1}{2}+c_{s} / v\right) X_{0}^{-}(1 / v, 0)} \\
& \times J\left(\lambda_{a}, \kappa_{0} ; \xi\right) .
\end{aligned}
$$

Correspondingly, as $t \rightarrow \infty$,

$$
\begin{aligned}
\sigma(\xi, t) \sim & \frac{\mathrm{e}^{-\lambda_{a} \xi} \Gamma\left(\kappa_{0}+c_{s} / v\right) \cos \pi \kappa_{0}\left(c_{s} t\right)^{-\kappa_{0}-1 / 2}}{\pi\left(v / c_{s}\right) \Gamma\left(\frac{1}{2}+c_{s} / v\right) \Gamma\left(\frac{1}{2}-\kappa_{0}\right) X_{0}^{-}(1 / v, 0)} \\
& \times J\left(\lambda_{a}, \kappa_{0} ; \xi\right) .
\end{aligned}
$$

The long-time behaviour of the stress singularity coefficient again follows by considering $\xi \rightarrow 0$. This gives

$\hat{k}(s) \sim \frac{c_{s}^{1 / 2-\kappa_{0}} \Gamma\left(\kappa_{0}+c_{s} / v\right) \cos \pi \kappa_{0} \Gamma\left(\frac{1}{2}-\kappa_{0}\right) s^{\kappa_{0}-1 / 2}}{\pi v \Gamma\left(\frac{1}{2}+c_{s} / v\right) X_{0}^{-}(1 / v, 0)}$

as $s \rightarrow 0$, and

$k(t) \sim \frac{\Gamma\left(\kappa_{0}+c_{s} / v\right) \cos \pi \kappa_{0}\left(c_{s} t\right)^{-\kappa_{0}-1 / 2}}{\pi\left(v / c_{s}\right) \Gamma\left(\frac{1}{2}+c_{s} / v\right) X_{0}^{-}(1 / v, 0)}$ as $t \rightarrow \infty$. It is noted that the formula (5.21) agrees with the result (4.8), because $\Gamma\left(\frac{1}{2}-\kappa_{0}\right) \Gamma\left(\frac{1}{2}+\kappa_{0}\right)=$ $\pi / \cos \pi \kappa_{0}$.

\subsection{The parameters $\lambda_{a}, \lambda_{b}$}

Recall that, in general, the zeros of the function $\alpha^{2}(\zeta, s)$ are called $a_{0}, a_{1}$ and $a_{2}$, where only $a_{0}$ is in $\mathscr{D}^{+}$, and then only when $v<c_{l}$. Elementary asymptotic analysis shows that, as $s \rightarrow 0$, the three zeros of $\alpha^{2}(\zeta, s)$ take the forms

$$
\begin{aligned}
& \zeta_{\mathrm{I}} \sim \frac{c_{l}^{2}-(1+f) v^{2}}{s v \tau\left(c_{l}^{2}-v^{2}\right)}, \\
& \zeta_{\mathrm{II}} \sim \frac{1}{v-(1+f)^{-1 / 2} c_{l}}, \\
& \zeta_{\text {III }} \sim \frac{1}{v+(1+f)^{-1 / 2} c_{l}} .
\end{aligned}
$$

Thus,

$a_{0} \sim \begin{cases}\zeta_{\mathrm{II}}, & 0 \leqslant v<(1+f)^{-1 / 2} c_{l}, \\ \zeta_{\mathrm{I}}, & (1+f)^{-1 / 2} c_{l}<v<c_{l}\end{cases}$

and hence

$$
\lambda_{a}= \begin{cases}0, & 0 \leqslant v<(1+f)^{-1 / 2} c_{l}, \\ \frac{(1+f) v^{2}-c_{l}^{2}}{v \tau\left(c_{l}^{2}-v^{2}\right)}, & (1+f)^{-1 / 2} c_{l}<v<c_{l} .\end{cases}
$$

The formulae for $\lambda_{b}$ are the same, except that $c_{s}$ replaces $c_{l}$. It should be noted that the speeds $c_{l} /(1+f)^{1 / 2}, c_{s} /(1+f)^{1 / 2}$ are the phase speeds of low-frequency longitudinal and shear waves, corresponding to the relaxed moduli.

\subsection{The traction for $t \rightarrow \infty$ and large $\xi$}

To describe the behaviour of the traction $\sigma(\xi, t)=\sigma_{12}\left(x_{1}-v t, 0, t\right)$ as $t \rightarrow \infty$ with $\xi$ large, introduce the constants

$$
\begin{aligned}
C_{0} & =\frac{\sqrt{c_{s}} \Gamma\left(\frac{1}{2}+c_{s} / v\right)}{\pi v \Gamma\left(1+c_{s} / v\right) X_{0}^{-}(1 / v, 0)}, \\
C_{1} & =-\frac{4 \eta_{s} \eta_{l}}{R(v)} C_{0}, \\
C_{2} & =\frac{\Gamma\left(\kappa_{0}+c_{s} / v\right) \cos \pi \kappa_{0} c_{s}^{1 / 2-\kappa_{0}}}{\pi v \Gamma\left(\frac{1}{2}+c_{s} / v\right) \Gamma\left(\frac{1}{2}-\kappa_{0}\right) X_{0}^{-}(1 / v, 0)}
\end{aligned}
$$


and analyse formulae (5.13) and (5.20) when $\xi \gg c_{s} \tau .{ }^{3}$ We state the final result as follows:

If the crack propagates at a sub-Rayleigh speed $v<c_{\mathrm{R}}$, and $t \rightarrow \infty$, then the shear traction ahead of the crack is not exponentially damped:

$\sigma(\xi, t) \sim \frac{C}{(1+f)^{2}}(\xi t)^{-1 / 2}, \quad \xi \gg c_{s} \tau$,

$C= \begin{cases}C_{0}, & 0<v<(1+f)^{-1 / 2} c_{s} \\ C_{1}, & (1+f)^{-1 / 2} c_{s}<v<c_{\mathrm{R}}\end{cases}$

unless $c_{\mathrm{R}}>(1+f)^{-1 / 2} c_{l}$, and $v \in\left((1+f)^{-1 / 2} c_{l}\right.$, $\left.c_{\mathrm{R}}\right)$ when the traction decays exponentially with respect to $\xi$ :

$\sigma(\xi, t) \sim\left(\frac{v \tau \lambda_{a}+1}{v \tau \lambda_{a}+1+f}\right)^{2} \frac{C_{1}}{\left(\pi \lambda_{a} t\right)^{1 / 2} \xi} \mathrm{e}^{-\lambda_{a} \xi}, \quad \xi \gg c_{s} \tau$.

For the transonic case, if $c_{s}>(1+f)^{-1 / 2} c_{l}$, then the shear traction vanishes at infinity exponentially:

$\sigma(\xi, t) \sim\left(\frac{v \tau \lambda_{a}+1}{v \tau \lambda_{a}+1+f}\right)^{2} \frac{C_{2}}{\left(\lambda_{a} t\right)^{\kappa_{0}+1 / 2} \xi} \mathrm{e}^{-\lambda_{a} \xi}, \quad \xi \gg c_{s} \tau$.

Otherwise, the traction has the exponential decay (5.29) if $(1+f)^{-1 / 2} c_{l}<v<c_{l}$ only. For the lower speeds, $v \in\left(c_{s},(1+f)^{-1 / 2} c_{l}\right)$, exponential decay is not observed and the behaviour of the traction for large $\xi$ is described by

$\sigma(x, t) \sim \frac{\Gamma\left(\frac{1}{2}-\kappa_{0}\right)}{(1+f)^{2}} C_{2} \xi^{\kappa_{0}-1 / 2} t^{-\kappa_{0}-1 / 2}, \quad \xi \gg c_{s} \tau$.

\section{Concluding remarks}

The problem of a dynamically-advancing crack in a viscoelastic medium, subjected to a particular

\footnotetext{
${ }^{3}$ Note, however, that since the limit $t \rightarrow \infty$ was taken first, the expressions that result are subject to the restriction $\xi \ll c_{s} t$, so that they apply far behind the wavefronts.
}

loading on the faces of the crack, has been solved. It generalises to viscoelasticity a recent solution obtained for an elastic medium by Huang and Gao (2001), the novelty of which was the study of the transonic range of crack speeds. It is seen, as expected, that the viscoelastic dissipation reduces the stress ahead of the crack, in comparison with its values in an elastic medium. A feature which ran counter to our expectations is that the strengths of the stress singularities decay only algebraically as time increases, though their actual magnitudes are less than in the elastic case. However, a study of the long-time form of the stress as a function of position $\xi$ ahead of the crack tip shows exponential decay, for some speed ranges: for sub-Rayleigh propagation, it is necessary that both $\lambda_{a}$ and $\lambda_{b}$ should be strictly negative. Otherwise, exponential decay is not observed, though there is still a transition in behaviour as $v$ increases past $c_{s} /(1+f)^{1 / 2}$, because the term associated with $\sigma_{b}$ starts to display exponential decay. For transonic propagation, $\lambda_{a}$ is the only relevant parameter. The exact range of possibilities depends on the relative magnitudes of $c_{\mathrm{R}}, c_{s} /(1+f)^{1 / 2}, c_{s}, c_{l} /(1+$ $f)^{1 / 2}$ and $c_{l}$.

Onset of exponential decay as crack speed $v$ increases through a "low-frequency" wave speed was first observed by Willis (1967) in a study of steady-state propagation under Mode III loading. A more complete study, including both steady state and more general transient loadings, also including transonic crack speeds, is in progress.

\section{Acknowledgement}

The support of the US Office of Naval Research through contract number N00014-01-1-0204 is gratefully acknowledged.

\section{Appendix A. Different bulk and shear relaxation}

We aim to show that the procedure based on solving a scalar Riemann-Hilbert problem is applicable for the more general case of the constitutive equation 
$\sigma_{i j}=K_{b} \delta_{i j} g_{1} * d e_{k k}+2 \mu g_{2} * d e_{i j}^{\prime}$,

where $K_{b}=\lambda+\frac{2}{3} \mu$ is the bulk modulus, $e_{i j}^{\prime}$ denotes the strain deviator $e_{i j}-\frac{1}{3} \delta_{i j} e_{k k}$, and $g_{1}(t)$ and $g_{2}(t)$ are the bulk and shear relaxation functions respectively:

$$
\begin{aligned}
g_{j}(t) & =\frac{1}{1+f_{j}}\left[1+f_{j} \exp \left(-\frac{1+f_{j}}{\tau_{j}} t\right)\right] H(t), \\
j & =1,2 .
\end{aligned}
$$

The parameters $f_{1}, f_{2}$ and $\tau_{1}, \tau_{2}$ are assumed to be different. Following the scheme of Section 2, instead of the functions (2.25), we get

$$
\begin{aligned}
& \alpha^{2}=-\zeta^{2}+\frac{\rho(v \zeta-1)^{2}}{K_{b} h_{1}+\frac{4 \mu}{3} h_{2}}, \\
& \beta^{2}=-\zeta^{2}+\frac{\rho(v \zeta-1)^{2}}{\mu h_{2}},
\end{aligned}
$$

where

$$
\begin{aligned}
& h_{j}(\zeta, s)=\frac{\zeta-\zeta_{0}^{(j)}}{\zeta-\zeta_{1}^{(j)}}, \\
& \zeta_{0}^{(j)}=\frac{\tau_{j}+1 / s}{v \tau_{j}}, \quad \zeta_{1}^{(j)}=\frac{\tau_{j}+(1+f) / s}{v \tau_{j}}, \\
& \quad j=1,2 .
\end{aligned}
$$

It is clear that as before, the function $\beta(\zeta, s)$ has four branch points in the $\zeta$-plane, whereas the function $\alpha$ has six branch points. We choose those branches of the functions $\alpha, \beta$ which meet the conditions: $\operatorname{Re}(\alpha)>0, \operatorname{Re}(\beta)>0$ for all $\zeta \in L$.

Use of the boundary conditions gives the Riemann-Hilbert boundary-value problem (3.4) with the function $K(\zeta, s)$ defined by

$K(\zeta, s)=-\frac{\zeta\left(\alpha^{2}+\zeta^{2}\right) \beta(\zeta, s)\left[K_{b} h_{1}(\zeta, s)+\frac{4 \mu}{3} h_{2}(\zeta, s)\right]}{\mu^{2} h_{2}^{2}(\zeta, s) D(\zeta, s)}$,

with

$$
\begin{gathered}
D(\zeta, s)=4 \zeta^{2} \alpha \beta+\frac{\beta^{2}-\zeta^{2}}{\mu h_{2}}\left[K_{b}\left(\alpha^{2}+\zeta^{2}\right) h_{1}\right. \\
\left.+\frac{2 \mu}{3}\left(2 \alpha^{2}-\zeta^{2}\right) h_{2}\right] .
\end{gathered}
$$

The new problem can be also solved by the method of Section 3.

Appendix B. A computationally convenient form for $X_{0}^{-}(\mathbf{1} / v, s)$

Consider first the case of subsonic propagation. We notice that $1 / v \notin L=\{\operatorname{Im}(s)=-\delta,-\infty<$ $\operatorname{Re}(s)<\infty\} \quad(\delta>0)$, and therefore, the integral Eq. (3.16) defining $\log X_{0}^{-}(1 / v, s)$ is not singular. For numerical purposes, it is convenient to transform the integral to one around the unit circle:

$$
\begin{aligned}
& X_{0}^{-}\left(\frac{1}{v}, s\right) \\
& =\exp \left\{\frac{1}{\pi \mathrm{i}} \int_{|w|=1} \frac{H(w, s) \mathrm{d} w}{-\delta-1+(-\delta+1) w-\frac{1}{v}(w+1)}\right\},
\end{aligned}
$$

where

$$
\begin{aligned}
H(w, s)= & \frac{1}{w+1} \\
& \times \log K_{0}\left(\frac{-\delta-1+(-\delta+1) w}{w+1}, s\right) .
\end{aligned}
$$

The function $K_{0}(\eta, s)$ is continuous at infinity, and $K_{0}(\eta, s)=1+O\left(\eta^{-1}\right), \quad \eta \rightarrow-\delta \pm \mathrm{i} \infty$. Therefore, the new function is bounded at $w=-1: H(w, s)=$ $O(1), w \rightarrow-1$. Because of the sensitivity to errors in the values of the function $H(w, s)$ in a neighbourhood of the point $w=-1$, it is important to know the value $H(-1, s)$. To find it we expand out the functions

$$
\begin{gathered}
\alpha(\zeta, s)=-\zeta \operatorname{i} \eta_{l} \operatorname{sgn} \operatorname{Im}(\zeta)\left[1+\frac{v}{c_{l}^{2} \eta_{l}^{2}} \frac{1+(2 \tau s)^{-1} f}{\zeta}\right. \\
\left.+O\left(\frac{1}{\zeta^{2}}\right)\right]
\end{gathered}
$$

$$
\begin{aligned}
\beta(\zeta, s)=-\zeta \operatorname{i} \eta_{s} \operatorname{sgn} \operatorname{Im}(\zeta)\left[1+\frac{v}{c_{s}^{2} \eta_{s}^{2}} \frac{1+(2 \tau s)^{-1} f}{\zeta}\right. \\
\left.+O\left(\frac{1}{\zeta^{2}}\right)\right], \quad \zeta \rightarrow-\delta \pm \mathrm{i} \infty .
\end{aligned}
$$


Therefore, the Rayleigh function $D(\zeta, s)$ has asymptotic behaviour as follows:

$$
\begin{aligned}
D(\zeta, s)= & \zeta^{4} R(v)+\zeta^{3} \frac{4 v\left(1+\{2 \tau s\}^{-1} f\right)}{c_{s}^{2}} \\
& \times\left(2-\frac{v^{2}}{c_{s}^{2}}-\frac{c_{s}^{2} \eta_{s}^{2}+c_{l}^{2} \eta_{l}^{2}}{c_{l}^{2} \eta_{l} \eta_{s}}\right)+O\left(\zeta^{2}\right), \\
\zeta \rightarrow- & \delta \pm \mathrm{i} \infty .
\end{aligned}
$$

Substituting formulae (B.3), (B.4) into (3.5) gives the first two terms of the asymptotic expansion for the function $K(\zeta, s)$

$$
\begin{aligned}
& K(\zeta, s)=\mathrm{i} \kappa \operatorname{sgn} \operatorname{Im}(\zeta)\left[1-\frac{\Lambda(s)}{\zeta}+O\left(\frac{1}{\zeta^{2}}\right)\right], \\
& \zeta \rightarrow-\delta \pm \mathrm{i} \infty
\end{aligned}
$$

where

$$
\begin{aligned}
\Lambda(s)=\frac{f}{v \tau s} & +\left(1+\frac{f}{2 \tau s}\right)\left[\frac{2}{v}-\frac{v}{c_{s}^{2} \eta_{s}^{2}}\right. \\
& \left.+\frac{4 v}{c_{s}^{2} R(v)}\left(2-\frac{v^{2}}{c_{s}^{2}}-\frac{c_{s}^{2} \eta_{s}^{2}+c_{l}^{2} \eta_{l}^{2}}{c_{l}^{2} \eta_{l} \eta_{s}}\right)\right] .
\end{aligned}
$$

Therefore,

$$
\begin{aligned}
K_{0}(\zeta, s) & =\frac{K(\zeta, s)}{\kappa \tan \pi c_{s} \zeta} \\
& =1-\frac{\Lambda(s)}{\zeta}+O\left(\frac{1}{\zeta^{2}}\right), \quad \zeta \rightarrow-\delta \pm \mathrm{i} \infty .
\end{aligned}
$$

Using the definition (B.2), it follows that the asymptotic form of $H(w, s)$ as $w \rightarrow-1$ is given by

$H(w, s)=\frac{1}{2} \Lambda(s)+O(w+1), \quad w \rightarrow-1$.

Thus, the function $H(w, s)$ is bounded and continuous everywhere on the unit circle $|w|=1$.

In the case of transonic propagation, we again convert the Cauchy integral (3.16) into the integral (B.1) around the unit circle and analyse the behaviour of the function $H(w, s)$ as $w \rightarrow-1$. The function $\alpha(\zeta, s)$ has the same asymptotic expansion as in the subsonic case, namely, (B.3). For the function $\beta(\zeta, s)$,

$$
\begin{gathered}
\beta(\zeta, s)=-\zeta \eta_{s}\left[1+\frac{v}{c_{s}^{2} \eta_{s}^{2}} \frac{1+(2 \tau s)^{-1} f}{\zeta}+O\left(\frac{1}{\zeta^{2}}\right)\right], \\
\zeta \rightarrow-\delta \pm \mathrm{i} \infty .
\end{gathered}
$$

By applying the same argument as in the subsonic case, we finally get

$$
\begin{array}{r}
K(\zeta, s)=\frac{\kappa}{\cos \pi \kappa_{0}\left( \pm \mathrm{i}+\tan \pi \kappa_{0}\right)}\left[1-\frac{\Lambda^{ \pm}(s)}{\zeta}\right. \\
\left.+O\left(\frac{1}{\zeta^{2}}\right)\right], \quad \zeta \rightarrow-\delta \pm \mathrm{i} \infty
\end{array}
$$

where

$$
\begin{aligned}
\Lambda^{ \pm}(s)= & \frac{f}{v \tau s}+\left(1+\frac{f}{2 \tau s}\right) \\
& \times\left[\frac{2}{v}-\frac{v}{c_{s}^{2} \eta_{s}^{2}}+\frac{v}{c_{s}^{2} \eta_{s} \eta_{l}\left( \pm \mathrm{i}+\tan \pi \kappa_{0}\right)}\right. \\
& \left.\times\left(2-\frac{v^{2}}{c_{s}^{2}} \pm \mathrm{i} \frac{c_{s}^{2} \eta_{s}^{2}+c_{l}^{2} \eta_{l}^{2}}{c_{l}^{2} \eta_{l} \eta_{s}}\right)\right] .
\end{aligned}
$$

It becomes evident that although the function $H(w, s)$ is bounded at $w=-1$, it is discontinuous at this point:

$$
\begin{aligned}
& H(w, s)=\frac{1}{2} \Lambda^{ \pm}(s)+O(w+1), \\
& w=\mathrm{e}^{\mathrm{i} \theta}, \quad \theta \rightarrow \pm \pi \mp 0 .
\end{aligned}
$$

\section{References}

Abate, J., Whitt, W., 1995. Numerical inversion of Laplace transforms of probability distributions. ORSA J. Comput. 7, 36-43.

Antipov, Y.A., Arutyunyan, N.K., 1991. Contact problems of the theory of elasticity with friction and adhesion. Appl. Math. Mech. 55, 887-901.

Broberg, K.B., 1989. The near-tip field at high crack velocities. Int. J. Fract. 39, 1-13.

Freund, L.B., 1972. Crack propagation in an elastic solid subjected to general loading - I. Constant rate of extension. J. Mech. Phys. Solids 20, 129-140. 
Gakhov, F.D., 1966. Boundary Value Problems. Pergamon Press, Oxford.

Huang, Y., Gao, H., 2001. Intersonic crack propagation. Part I: The fundamental solution. ASME J. Appl. Mech. 68, 169175.

Noble, B., 1988. Methods based on the Wiener-Hopf technique. Chelsea Publishing Company, New York.

Nuller, B.M., 1976. Contact problem for an elastic wedge reinforced by a bar of uniform resistance. Sov. Phys. Dokl. 20, 789-790.

Rosakis, A.J., Samudrala, O., Coker, D., 1999. Cracks faster than the shear wave speed. Nature 284, 1337-1340.

Walton, J.R., 1990. The dynamic energy release rate for a steadily propagating mode I crack in an infinite, linearly viscoelastic body. ASME J. Appl. Mech. 57, 343353.

Herrmann, J.M., Walton, J.R., 1994. On the energy release rate for dynamic transient mode I crack propagation in a general linear viscoelastic body. Quart. Appl. Math. 52, 201-228.

Willis, J.R., 1967. Crack propagation in viscoelastic media. J. Mech. Phys. Solids 15, 229-240.

Willis, J.R., 1997. Asymptotic analysis in fracture, Advances in Fracture Research. In: Karihaloo, B.L., Mai, Y.-W., Ripley, M.I., Ritchie, R.O. (Eds.), Proceedings ICF9, vol. 4: Theoretical and Computational Directions. Pergamon, Oxford, pp. 1849-1859.

Willis, J.R., 2000. Asymptotic analysis in fracture: an update. Int. J. Fract. 100, 85-103. 